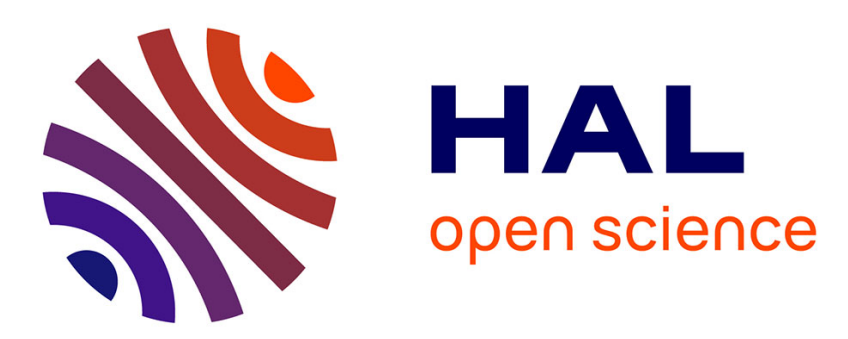

\title{
Elaboration du rendement et fertilisation azotée du blé d'hiver en Champagne crayeuse III. - Influence des variations pédologiques; conséquences pour la conduite de la culture
}

Jean Boiffin, Jean-Marc Meynard, Michel Sebillotte, Jacques Caneill

\section{To cite this version:}

Jean Boiffin, Jean-Marc Meynard, Michel Sebillotte, Jacques Caneill. Elaboration du rendement et fertilisation azotée du blé d'hiver en Champagne crayeuse III. - Influence des variations pédologiques ; conséquences pour la conduite de la culture. Agronomie, 1982, 2 (5), pp.417-428. hal-00884400

\author{
HAL Id: hal-00884400 \\ https://hal.science/hal-00884400
}

Submitted on 1 Jan 1982

HAL is a multi-disciplinary open access archive for the deposit and dissemination of scientific research documents, whether they are published or not. The documents may come from teaching and research institutions in France or abroad, or from public or private research centers.
L'archive ouverte pluridisciplinaire HAL, est destinée au dépôt et à la diffusion de documents scientifiques de niveau recherche, publiés ou non, émanant des établissements d'enseignement et de recherche français ou étrangers, des laboratoires publics ou privés. 


\title{
Elaboration du rendement et fertilisation azotée du blé d'hiver en Champagne crayeuse
} III. - Influence des variations pédologiques ; conséquen-
ces pour la conduite de la culture

\author{
Jcan BOIFFIN, Jean-Marc MEYNARD, Michel SEBILLOTTE \& Jacques CANEILL
}

I.N.R.A., Chaire d'Agronomie, Institut National Agronomique Paris-Grignon, 16, rue Claude-Bernard, F 75231 Paris Cedex 05.

\section{RÉSUMÉ}

Blé d'hiver, Elaboration du rendement,

Azote,

Fertilisation azotée, Profil pédologique, Classement des sols, Sols de craie, Conseil technique.
Dans 2 mémoires précédents, on a exposé un protocole d'étude de la culture du blé en Champagne craycuse ct discuté le domaine de validité de la méthode du bilan prévisionnel.

On cherche ici à préciser, au niveau de la parcelle, les modalités de conduite de la culture, dont celles de fumure azotée. Dans une région où les sols sont très variés malgré l'homogénéité de substrat géologique, il a fallu analyser les conséquences de cette variabilité sur l'élaboration du rendement du blé.

Dans une première partie, on décrit les 112 stations étudiées, qui se répartissent en 11 catégories élémentaires distinguées selon la morphologie du profil (tabl. 1). Les principaux traits de la variaoilité entre profils sont les différences de régime thermique entre les «Terres blanches » et les «Terres rouges » (tabl. 3) et les variations de profondeurs d'enracinement, dont résultent entre autres des différences de réserves hydriques (tabl. 4). Dans une deuxième partie, on étudie les conséquences de cette variabilité pédologique sur la croissance du blé d'hiver. On montre que le paramètre «Profondeur d'enracinement» est déterminant pour comprendre l'utilisation par la culture de l'azote minéral présent dans le sol en sortie d'hiver (fig. 1). On met en évidence l'effet limitant sur la croissance du régime thermique défavorable des Terres blanches (fig. 2). On en discute les conséquences pour le fractionnement et les dates d'apport des engrais azotés. Enfin, on analyse l'influence du profil sur l'alimentation hydrique et le rendement potentiel de la culture (fig. 3 et 4 ).

Dans la troisième partie, on propose un regroupement des unités pédologiques de base, correspondant à des catégories homogènes vis-à-vis du comportement de la culture, et donc du conseil technique (tabl. 6).

\section{SUMMARY}

Winter wheat,

Yield formation proces-

ses,

Nitrogen,

Nitrogen fertilization,

Soil profile,

Soil classification,

Chalky soils,

Technical advice.
Yield elaboration and nitrogen fertilization of winter wheat in Champagne crayeuse - III. - Effect of pedological variations; consequences for crop management

In two previous papers, we presented the experimental design and methods chosen to study winter wheat in Champagne crayeuse, and discussed the range of applicability of the predictive balance method for determining nitrogen fertilization rates.

In this paper, we attempt to define the basis of crop management at the plot scale, including nitrogen fertilization. In a region where soils are very different, although over the same geological substratum, we had to analyse the effects of this variability on the yield elaboration process.

In the first part, we describe the 112 plots studied, which are distributed within 11 types according to their profile morphology (table 1). The main differences between the soil-types concern (a) temperature conditions (table 3) and (b) root depth and consequent variations in available water content.

In the second part, we analyse the consequences of this pedological variability on the growth of winter wheat. Root depth is critical to explain the response of winter wheat to the mineral nitrogen content remaining in the soil in late winter (fig. 1). The growth-limiting effect of low temperatures in the "white soils" class is shown. Consequences for timing and split application of nitrogen fertilizers are discussed. We also analyse water supply and potential yield as influenced by the soil profile (fig. 3 and 4 ).

In the third part, we propose a classification regrouping the elementary soil types in land-use classes, homogeneous for crop behaviour and accordingly, for advisory purposes (table 6).

\section{INTRODUCTION}

Ayant conçu et mis en æuvre un protocole d'enquête et d'expérimentation axé sur l'étude de la fertilisation azotée du blé (BolfFin et al., 1981), nous avons conclu que la méthode du bilan prévisionnel (HÉBERT, 1969 ; RÉMY \& HÉBERT, 1977) pouvait être, sous certaines conditions, employée en Champagne crayeuse pour déterminer les doses d'engrais azoté (MEYNARD et al., 1981). Ce résultat constitue en soi une contribution importante à l'effort qui se 
développe actuellement en France pour rationaliser la culture du blé.

Cependant, la validité de principe de la méthode du bilan prévisionnel étant établie, restent à préciser ses modalités d'application au niveau de la parcelle agricole. Dans une petite région telle que la Champagne crayeuse, une des principales sources de l'hétérogénéité entre parcelles est la variabilité pédologique ; ce mémoire se propose d'en analyser les répercussions sur la culture et sa conduite.

Dans une $1^{\mathrm{re}}$ partie, on décrira la variabilité du milieu, en insistant sur les caractères analytiques ou synthétiques considérés par l'agronome comme déterminants pour la croissance du blé.

Dans la $2^{\mathrm{e}}$ partie, on étudiera l'influence des caractères ainsi retenus sur les processus d'élaboration du rendement.

Cette analyse débouchera, dans une $3^{\mathrm{e}}$ partie, sur un regroupement des unités pédologiques de base, correspondant à des catégories homogènes vis-à-vis du conseil technique.

\section{PROTOCOLE EXPÉRIMENTAL}

Ce protocole a été exposé de façon détaillée dans le premier mémoire de cette série consacrée à l'élaboration du rendement et à la fertilisation azotée du blé en Champagne crayeuse (BOIFFIN et al., 1981).

Des stations d'enquête ( "parcelles-types »), répartics en couples dont les 2 membres se situent au sein de la même parcelle culturale, permettent de comparer la végétation sur 2 types de sols différents (« Terre blanche» et «Terre rouge »). Sur chaque station sont appliquées les doses d'engrais azoté calculées d'après le bilan prévisionnel (dose globale X, dose tallage Y). En 1977, est accolé à chaque station un témoin sans azote.

Par ailleurs, en 1977, le dispositif d'enquête est complété par 2 essais de fumure azotée réalisés dans 2 zones de la même parcelle, l'un en Terre blanche (essai EEB), l'autre en Terre rouge (essai EER). Leur protocole est exposé dans les mémoires précédents déjà cités.

\section{DESCRIPTION DU MILIEU}

\section{A. Climat}

Les caractéristiques climatiques des 3 principales campagnes d'étude (1974-75, 75-76, 76-77) ont été présentées dans le $1^{\text {er }}$ mémoire de cette série (BOIFFIN et al., 1981).

Les campagnes 1975 et 1977 ont des climats voisins, l'alimentation hydrique n'ayant pas limité la croissance avant la floraison.

En 1976, au contraire, la sécheresse précoce a perturbé l'absorption des engrais azotés et limité directement la croissance au cours de la montaison (SEBILlOTTE et al., 1978).

\section{B. Terrains}

\section{Généralités}

DUTIL \& BALLIF (1968) rappellent que la Champagne crayeuse est caractériséc par l'" affleurement d'un substratum calcaire de Crétacé supérieur (étage Sénonien), avec le faciès particulier de la craie, matériau tendre, très fortement carbonaté, poreux et toujours fissuré ». Ces mêmes auteurs décrivent la principale formation pédologique de la région, qui est une rendzine développée sur paléosol cryoturbé. Ce sol se caractérise par la présence de poches remplies de gravillons, en général recimentés. Entre les poches et sous celles-ci, on trouve des éléments grossiers de craie remaniée. Ceux-ci reposent sur la craie en place, fissurée. Selon sa position sur la pente, le paléosol peut avoir été plus ou moins tronqué par l'érosion et (ou) recouvert par des colluvions (souvent gravillonneuses, mais parfois de granulométrie plus fine). Enfin, il existe aussi des sols formés sur graveluches, substrat épais à forte dominance de graviers crayeux. A cette diversité de roches-mères s'ajoutent des variations d'intensité de l'évolution pédogénétique. En définitive, on rencontre les formations pédologiques suivantes (DuTIL, 1970 ; DuRAND, 1972):

- des rendzines grises directement sur craie, assez rares;

- des rendzines brunes, brun-rouges, sur paléosol cryoturbé et sur formations colluviales;

- des rendzines brunes sur graveluches;

- des sols bruns calcaires sur colluvions.

Par contre, tout en étant sensibles à la grande hétérogénéité (souvent intraparcellaire) des sols, les agriculteurs ne distinguent couramment que 2 grands types de terrains, désignés par les appellations vernaculaires «Terres blanches " et "Terres rouges". Dès le début de l'étude, il est apparu que le recoupement entre ces appellations et les familles pédologiques précédemment citées était imparfait. Par exemple, certains sols nettement différenciés par un même agriculteur correspondaient à la même catégorie « rendzine brune sur paléosol cryoturbé »; réciproquement, le passage d'une rendzine brune à une rendzine grise n'était pas toujours perçu comme un changement de comportement du terrain.

Il était donc nécessaire de procéder à une description relativement fine des sols des stations étudiées, de façon à établir, sans a priori, les relations entre le comportement agronomique et les caractéristiques permanentes du sol, et à ne regrouper les unités pédologiques de base qu'au terme de cette analyse.

\section{Présentation de l'échantillon des stations observées}

Au cours des 3 campagnes d'étude, 112 stations ont été décrites selon les procédures indiquées précédemment (Boiffin et al., 1981).

a) Caractérisation morphologique des profils et appellations vernaculaires

Chaque profil observé est rattaché à un "profil-type " défini, indépendamment de son interprétation pédogénétique, par la succession des matériaux observés sur une profondeur de 1,20 m. Cette caractérisation a été mise au point pour une large part grâce à des tournées d'observation avec les pédologues de la station de Châlons-sur-Marne. Les «profils-types» ainsi distingués correspondent sensiblement à la notion de série.

L'inventaire des stations observées est présenté dans le tableau 1, où l'on a fait apparaître la correspondance entre la succession des matériaux rencontrés au sein des profilstypes et les appellations vernaculaires des parcelles suivies. On constate :

— que la dénomination «Terre blanche "s"applique très généralement à des sols dont la couche arable est au contact des éléments grossiers de la craie, qu'il s'agisse de craie en 
TABLEAU 1

Inventaire des profils observés.

Review of the profiles described.

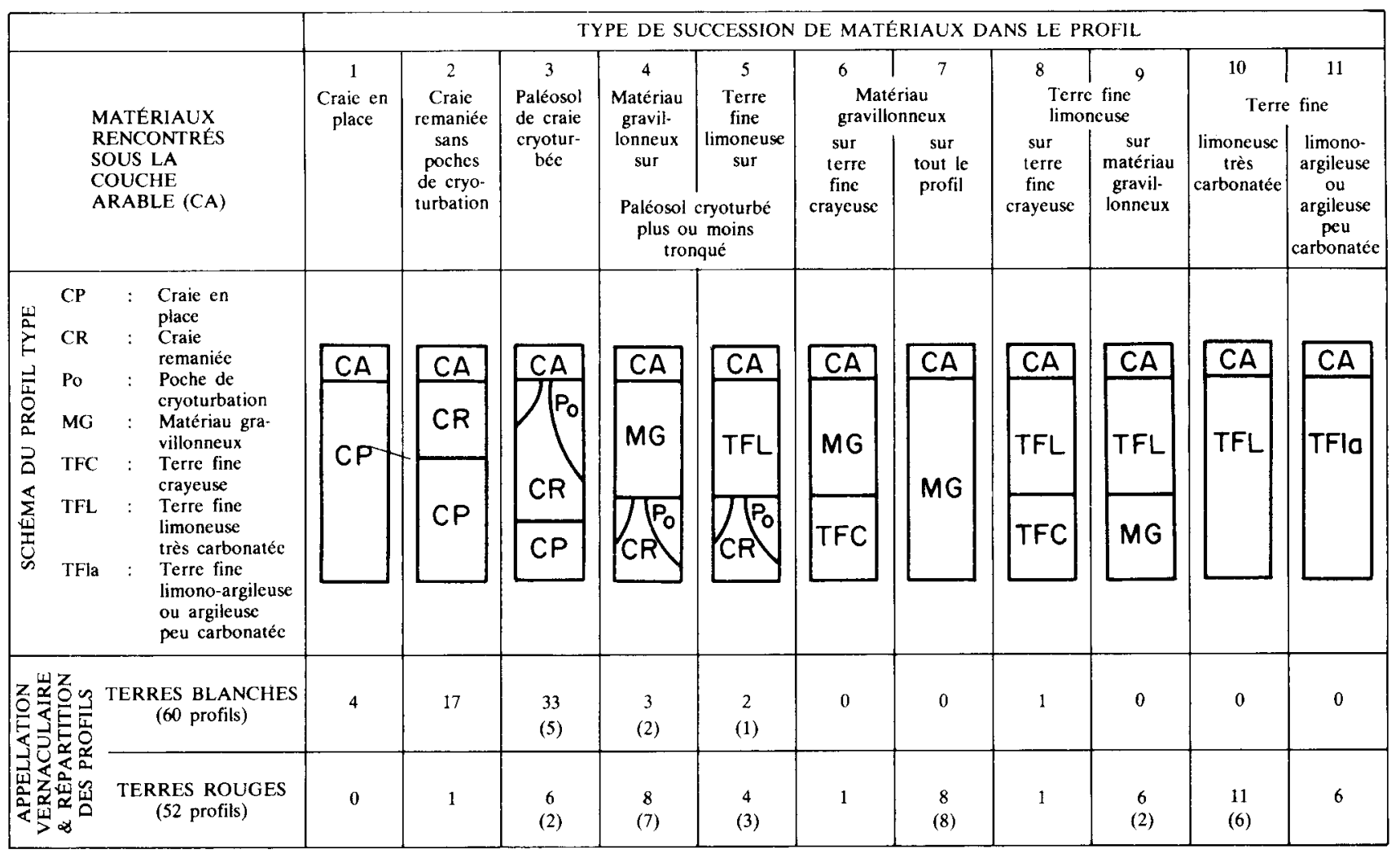

* Entre parenthèses, nombre de profils présentant un horizon massif cimenté ou un encroûtement.

place, de craie remaniée ou de «cheminée » séparant les poches de cryoturbation.

- que la dénomination "Terre rouge » s'applique à des sols beaucoup plus variés, où apparaissent divers matériaux d'origine principalement colluviale. Le point commun de ces profils est que la couche arable n'est pas en général, en contact avec la craie.

Une différenciation plus ou moins fréquente au sein de chaque catégorie, très importante sur le plan agronomique, concerne la présence éventuelle d'horizons indurés très compacts interdisant la pénétration des racines. Ces obstacles sont toujours observés à moins de $70 \mathrm{~cm}$ de profondeur. Il s'agit soit de véritables encroûtements de calcite, disposés en dalles horizontales, soit d'horizons à structure massive colmatés par des éléments fins formant un ciment diffus (c'est le cas, entre autres, de nombreuses poches de cryoturbation). Ce phénomène a récemment été décrit et analysé par DURAND (1980). Dans le tableau 1, on a signalé, entre parenthèses, le nombre de profils présentant, de ce fait, une limite horizontale continue infranchissable par les racines. On voit que ces cas sont particulièrement fréquents en Terre rouge, surtout sur colluvions gravillonneux.

\section{b) Caractères analytiques de la couche arable}

Le tableau 2 donne une présentation succincte des déterminations analytiques effectuées systématiquement sur chaque station. Les analyses granulométriques n'ont pas été effectuées, en raison des risques de destruction artificielle des fragments crayeux au cours de la dispersion (DURAND, 1972).
Les Terres blanches sont plus riches en calcaire et en éléments grossiers que les Terres rouges, différence d'ailleurs systématiquement vérifiée au sein des couples de stations. Il se confirme que les Terres rouges sont plus hétérogènes que les Terres blanches.

En revanche, les caractères plutôt liés à l'histoire culturale (matière organique, $\mathrm{P}, \mathrm{K}, \mathrm{Mg}$ assimilables) ne se distinguent pas d'un type vernaculaire à l'autre. Les teneurs en matière organique sont élevées en moyenne, mais très variables. Ces observations traduisent probablement le

\section{TABLEAU 2}

Résultats des analyses de la couche arable (en p. cent ou p. mille de la terre fine)

Moyenne de 106 résultats (entre parenthèses: écart-type).

Analytical results for the arable layer.

\begin{tabular}{lrlrr}
\hline \multicolumn{1}{c}{ Variable } & Terre blanche & \multicolumn{2}{c}{ Terre rouge } \\
\hline Matière organique (\%) & 3,46 & $(0,75)$ & 3,27 & $(0,94)$ \\
$\mathbf{N}($ KJELDHAL) (\%o) & 2,17 & $(0,32)$ & 2,07 & $(0,37)$ \\
$\mathrm{C} / \mathrm{N}$ & 9,26 & $(1,52)$ & 9,05 & $(1,29)$ \\
$\mathrm{CaCO}_{3}$ total $(\%)$ & 72,67 & $(9,81)$ & 48,88 & $(24,00)$ \\
$\begin{array}{l}\text { Eléments grossiers (>2 mm) } \\
(\%)\end{array}$ & 22,9 & $(7,32)$ & 15,8 & $(9,31)$ \\
$\begin{array}{l}\text { Acide phosphorique JoRET- } \\
\quad \text { HéBERT (\%) }\end{array}$ & 0,265 & $(0,071)$ & 0,205 & $(0,074)$ \\
Potassium échangeable (\%o) & 0,213 & $(0,083)$ & 0,227 & $(0,115)$ \\
Magnésium échangeable (\%) & 0,056 & $(0,014)$ & 0,069 & $(0,014)$ \\
\end{tabular}


faible taux de minéralisation de l'humus en sol très calcaire, ainsi que la variabilité assez importante des dates de défriche au sein de l'échantillon.

\section{c) Comportement thermique}

La faible vitesse de croissance des cultures de printemps sur les sols de craie à leur démarrage, est un fait d'observation courante, attribué à la lenteur du réchauffement des couches de surfaces. BALLIF \& DUTIL (1975), en couvrant le sol avec un mulch plastique, provoquent une augmentation notable de la température des couches superficielles et un accroissement spectaculaire de la vitesse de croissance du maïs.

En outre, on constate très généralement dans les parcelles hétérogènes, que les zones de terre plus colorée présentent en début de cycle, pour les cultures de printemps, une végétation plus vigoureuse et des stades de développement plus avancés. Il est raisonnable de supposer que ces hétérogénéités sont dues à des différences de régime thermique.

Pour le vérifier, on a implanté, en 1977, un dispositif de mesure des températures du sol sur les essais EEB et EER évoqués précédemment. On présente dans le tableau 3 les résultats des relevés du début du printemps. On constate bien une importante différence de réchauffement diurne des couches superficielles.

A la suite d'une étude de laboratoire, Roncin (1978) suggère que l'un des mécanismes déterminants de la lenteur de réchauffement des Terres blanches réside dans le fait que l'augmentation d'albedo (éclaircissement de surface) s'y produit avant réduction notable de l'évaporation. D'ailleurs, on peut constater qu'en sol nu, la surface des Terres blanches s'éclaircit plus vite que celle des Terres rouges lors du dessèchement. $\mathrm{Ce}$ comportement est probablement plus déterminant vis-à-vis de la dénomination vernaculaire que les différences de coloration, à sec ou à l'état humide, qui sont relativement faibles.

Une autre cause de différence dans le comportement thermique de surface pourrait provenir des différences d'humidité à la capacité au champ entre ces 2 catégories de sol: les plus fortes humidités des Terres blanches se traduisant par de plus fortes capacité calorifique et diffusivité thermique.

Sous ces hypothèses, une différence sensible de température entre Terre blanche et Terre rouge ne peut se manifester que lorsque le sol est nu ou peu couvert, en régime de réchauffement et lorsque le phénomène d'éclaircissement diurne se répète fréquemment. De telles conditions sont souvent réunies sous blé au printemps, jusqu'au début de la montaison.

\section{d) Réserves hydriques}

Sur ce point la diversité des profils observés est très importante. En effet, on constate :

- des différences considérables de profondeur d'enracinement, liées à la présence éventuelle d'obstacles structuraux à profondeur variable ;

- des variations de gamme d'eau utile des différents matériaux rencontrés allant de $20-25$ p. 100 d'humidité pour les éléments grossiers de craie enrobée de terre fine (GRAS \& MONNIER, 1963), à 10-12 p. 100 pour des horizons à prédominance de gravillons. Des observations d'évolution de profils hydriques sous blé (notamment en 1976), associées à quelques déterminations analytiques, aboutissent à des évaluations de la gamme d'eau utile pour chaque matériau rencontré, concordantes avec les données de BALLIF (1978, 1980). Ces évaluations sont fournies en annexe 1. Le tableau 4 présente les estimations de réserve utile, sur la profondeur d'enracinement, pour quelques profils caractéristiques. Tous les cas intermédiaires se présentent dans cette gamme de valeurs.

Cette variation importante des réserves calculées permet de comprendre pourquoi les Terres blanches permettent aux cultures de mieux résister à la sécheresse estivale. A cet aspect statique s'ajoutent les caractères particuliers concernant la circulation de l'eau dans la porosité de la craie, qui est de type ouvert et d'un diamètre homogène et relativement important, de l'ordre du micron (GRAS, 1972; PÉDRO, 1972; BALLIF, 1978): forte mobilité de l'eau non saturante et transferts capillaires en provenance de zones de craie non exploitées par les racines. En raison de ce dernier point, il est possible que des calculs tels que ceux du tableau 4 sous-estiment largement les réserves hydriques des sols où les racines accèdent à la craie, même sans y pénétrer profondément (cas de la station S2 où, en 1976, malgré un déficit climatique très élevé, un rendement supérieur à la moyenne a été obtenu). Ces remontées capillaires sont invoquées par BALLIF (1981) pour expliquer le maintien à des humidités relativement élevées des proches sous-sols de craie en période sèche sous culture de maïs, phénomène que nous avons également observé sous blé en 1976 (SEbillotte et al., 1978).

\section{TABLEAU 3}

Movenne des températures extrêmes sous blé. en Terre blanche et Terre rouge (essais EEB-EER, 1977) Average maximum and minimum temperatures in the soil under a wheat crop. T.B. : "White soil" - T.R. : "Red soil".

\begin{tabular}{|c|c|c|c|c|c|c|c|c|}
\hline & \multicolumn{4}{|c|}{ Températures maxima $\left({ }^{\circ} \mathrm{C}\right)$} & \multicolumn{4}{|c|}{ Températures minima $\left({ }^{\circ} \mathrm{C}\right)$} \\
\hline Profondeur & \multicolumn{2}{|c|}{$5 \mathrm{~cm}$} & \multicolumn{2}{|c|}{$15 \mathrm{~cm}$} & \multicolumn{2}{|c|}{$5 \mathrm{~cm}$} & \multicolumn{2}{|c|}{$15 \mathrm{~cm}$} \\
\hline Sol & $\mathrm{TB}$ & TR & $\mathrm{TB}$ & TR & TB & TR & TB & $\mathrm{TR}$ \\
\hline \multicolumn{9}{|l|}{ Décade: } \\
\hline $3^{\mathrm{e}}$ mars & 9,40 & 10,60 & 8,25 & 8,90 & 5,05 & 5,05 & 6,25 & 6,20 \\
\hline $1^{\text {er }}$ avril & 9,15 & 11,30 & 7,65 & 8,00 & 3,60 & 3,80 & 4,65 & 5,35 \\
\hline $2^{c}$ avril & 10,00 & 12,35 & 7,80 & 8,50 & 3,95 & 3,15 & 4,35 & 5,03 \\
\hline $3^{c}$ avril & 14,05 & 16,90 & 12,00 & 13,10 & 8,65 & 8,34 & 9,18 & 9,72 \\
\hline
\end{tabular}

TB : « Terre blanche» - TR : «Terre rouge » 
TABLEAU 4

Estimation des réserves utiles de quelques profils typiques.

Estimation of available water content for some typical soil profiles.

\begin{tabular}{|c|c|c|c|}
\hline $\begin{array}{l}\text { Station } \\
\text { observée }\end{array}$ & Type de profil & $\begin{array}{l}\text { Profondeur } \\
\text { d'enracinement } \\
\text { observée }(\mathrm{m})\end{array}$ & $\begin{array}{l}\text { R.U. } \\
\text { estimée } \\
(\mathrm{mm})\end{array}$ \\
\hline R 1 (1975) & Terre blanche sur craie remaniée sans poche (Colonne 2, Tab. 1) & 1 & 260 \\
\hline S 2 (1976) & $\begin{array}{l}\text { Terre rouge sur matériau gravillonneux sur paléosol cryoturbé } \\
\text { encroûté à } 40 \mathrm{~cm} \text { (Colonne } 4 \text { ) }\end{array}$ & 0,4 & 75 \\
\hline C 4 (1976) & $\begin{array}{l}\text { Terre rouge sur terre fine limoneuse ; tuf massif à } 70 \mathrm{~cm} \text { (Colonne } \\
\text { 10) }\end{array}$ & 0,7 & 150 \\
\hline Nr (1977) & $\begin{array}{l}\text { Terre rouge sur matériau gravillonneux encroûté à } 50 \mathrm{~cm} \\
\text { (Colonne } 7)\end{array}$ & 0,5 & 90 \\
\hline
\end{tabular}

\section{Conclusion}

L'analyse précédente nous amène à considérer que, sur le plan agronomique, les variations pédologiques en Champagne crayeuse se résument à 3 aspects essentiels :

- variations de profondeur du substrat crayeux ;

- présence éventuelle à profondeur variable d'obstacles structuraux à l'enracinement (dont certains sont sans doute aussi des obstacles à l'ascension capillaire) ;

- différences de comportement thermique des couches de surface.

Ces différences sont susceptibles d'intervenir en plusieurs points du schéma d'élaboration du rendement du blé (présenté dans BolfFin et al., 1981). On analysera successivement leur influence sur:

- l'exploitation par la culture du stock d'azote minéral du profil,

- la croissance en début de printemps et la valorisation des engrais azotés,

- l'alimentation hydrique de la culture.

\section{INFLUENCE DES VARIATIONS PÉDOLOGIQUES SUR L'ÉLABORATION DU RENDEMENT DU BLÉ}

\section{A. Influence sur le stock d'azote disponible}

La variabilité de la fourniture d'azote par le milieu peut être étudiée sur les témoins non fertilisés, ménagés sur chaque station en 1977 ; elle résulte de la somme :

- du reliquat minéral, mesuré en sortie d'hiver, et disponible à un instant donné sur la profondeur d'enracinement,

- de la minéralisation de l'humus et des résidus de récolte du précédent (betterave sur toutes les situations).

Le premier de ces 2 termes est assez variable, d'une part en raison de la fluctuation des pluies hivernales (DUTIL \& BALlif, 1971), mais surtout, dans le milieu considéré, en raison de la variation des profondeurs d'enracinement. Ainsi, en février 1977, la zone de concentration maximum de l'azote nitrique se trouve à environ $80 \mathrm{~cm}$ (MULLER, 1977), c'est-à-dire peu éloignée de la médiane des profondeurs d'enracinement à la floraison. Il en résulte que d'assez faibles variations de profondeur ont de fortes répercussions sur le reliquat disponible.
A l'inverse, en année de pluviométrie hivernale très supérieure à la normale, les reliquats disponibles sur la profondeur d'enracinement seraient plus faibles et beaucoup moins variables : d'une part les zones de concentration maximum sont nettement plus profondes que les racines, d'autre part, pour une même profondeur d'enracinement, la distribution statistique des reliquats est sensiblement lognormale et est donc moins variable pour de faibles valeurs (LiNDEMANN, 1977).

Au cours de la montaison, phase d'absorption intense d'azote, l'enracinement progresse en profondeur et accède à une part croissante des reliquats azotés. Parallèlement, se déterminent progressivement, en relation avec la nutrition azotée, les composantes du rendement.

Ainsi, on peut mettre logiquement en relation les reliquats azotés sur la profondeur d'enracinement atteinte respectivement au début de la montaison et à la floraison, avec d'une part le nombre d'épis, d'autre part le nombre de grains par unité de surface obtenus sur les témoins non fertilisés, c'est-à-dire lorsque l'azote est facteur limitant.

La figure 1 montre que, en l'absence de drainage après dosage des reliquats (condition vérifiée en 1977) les liaisons attendues sont bien constatées et sont étroites. Elles confirment l'influence déterminante de la variabilité des reliquats d'azote minéral en sortie d'hiver, déjà soulignée par de nombreux auteurs (entre autres HÉBERT, 1969 ; DuTIL \& BALLIF, 1971 ; GACHON, 1973). L'originalité des résultats présentés ici tient au fait que cette variabilité résulte de celle des substrats pédologiques et de ses conséquences sur l'enracinement. Ainsi, dans le milieu considéré, la méthode du bilan prévisionnel perdrait toute signification pratique si elle était appliquée en utilisant, pour le calcul des reliquats, une profondeur d'enracinement moyenne pour la région.

\section{B. Influence sur la croissance précoce et la valorisation des engrais azotés}

\section{Différence de comportement du blé en Terre blanche et en Terre rouge}

Sur les traitements fertilisés (doses $\mathrm{X}, \mathrm{Y}$ ) des parcellestypes, on constate que les nombres d'épis obtenus par unité de surface sont généralement plus élevés en Terre rouge qu'en Terre blanche (tabl. 5). Cette supériorité des Terres rouges concerne aussi d'autres caractères du peuplement résultant de la croissance au cours du tallage, comme la masse de matière sèche des parties aériennes par pied. En 1977, ce caractère a été mesuré sur un nombre suffisant de 


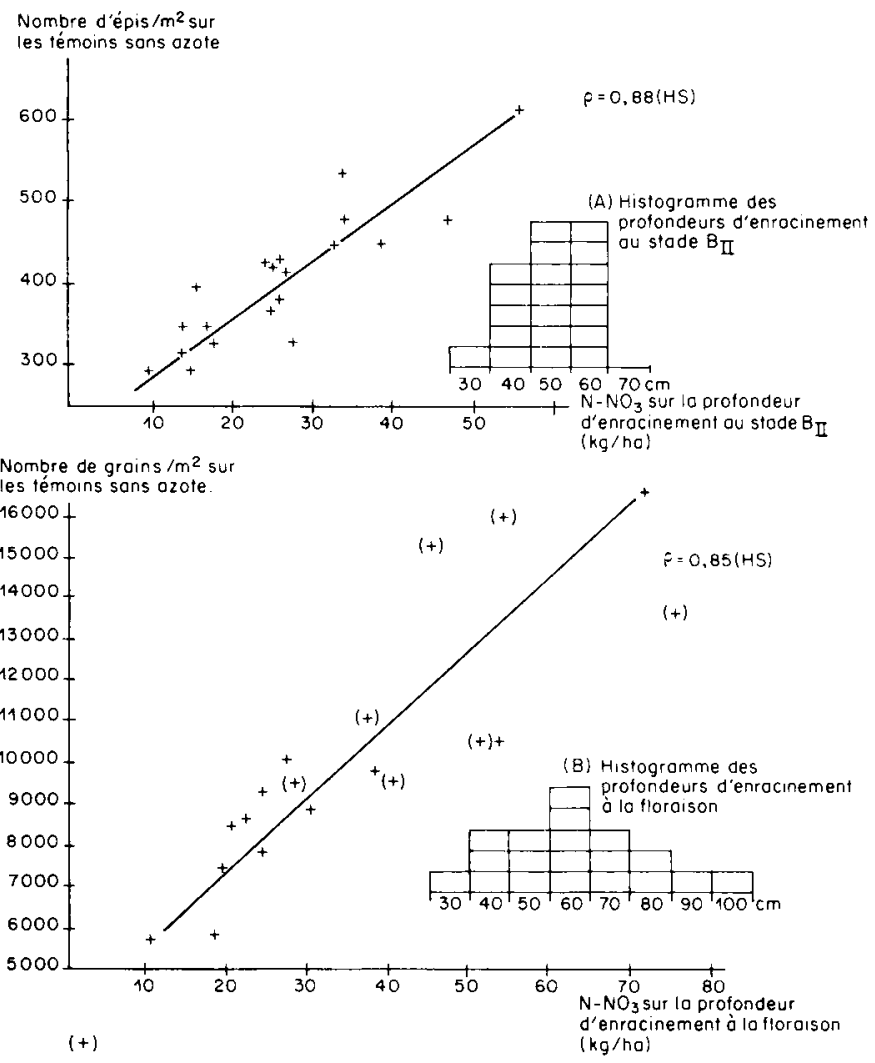

Figure 1

Composantes du rendement et dynamique de la fourniture d'azote par le milieu (tous terrains confondus, année 1977).

$(+)$ Les teneurs en nitrates du sol ayant été dosées jusqu'à $60 \mathrm{~cm}$, une extrapolation a été nécessaire pour évaluer le reliquat nitrique des 7 situations où l'enracinement dépassait cette profondeur à la floraison.

Yield components (ear and grain number per square meter) and nitrogen-supply pattern of the soil (all soil-types together, 1977); $(A)$ : histogram of root depth at the JONARD $B_{l l}$ stage; $(B)$ : histogram of root depth at anthesis.

(+) The nitrate nitrogen content was determined up to $60 \mathrm{~cm}$ depth, nitrogen content at greater depth being extrapolated on the 7 plots indexed.

couples; la différence moyenne est de $19 \mathrm{mg} / \mathrm{pied}$, soit 8 p. 100 , à l'avantage des stations de Terre rouge.
2. Analyse de l'influence du type de sol sur la réponse à l'azote au cours du tallage

Les essais EEB et EER permettent de mettre en évidence l'influence du type de sol sur les réponses à la fertilisation azotée ( $1^{\text {er }}$ apport) de la croissance des parties aériennes et de l'absorption d'azote, mesurées au stade $\mathrm{B}_{\mathrm{II}}$ (fig. $2 a$ ).
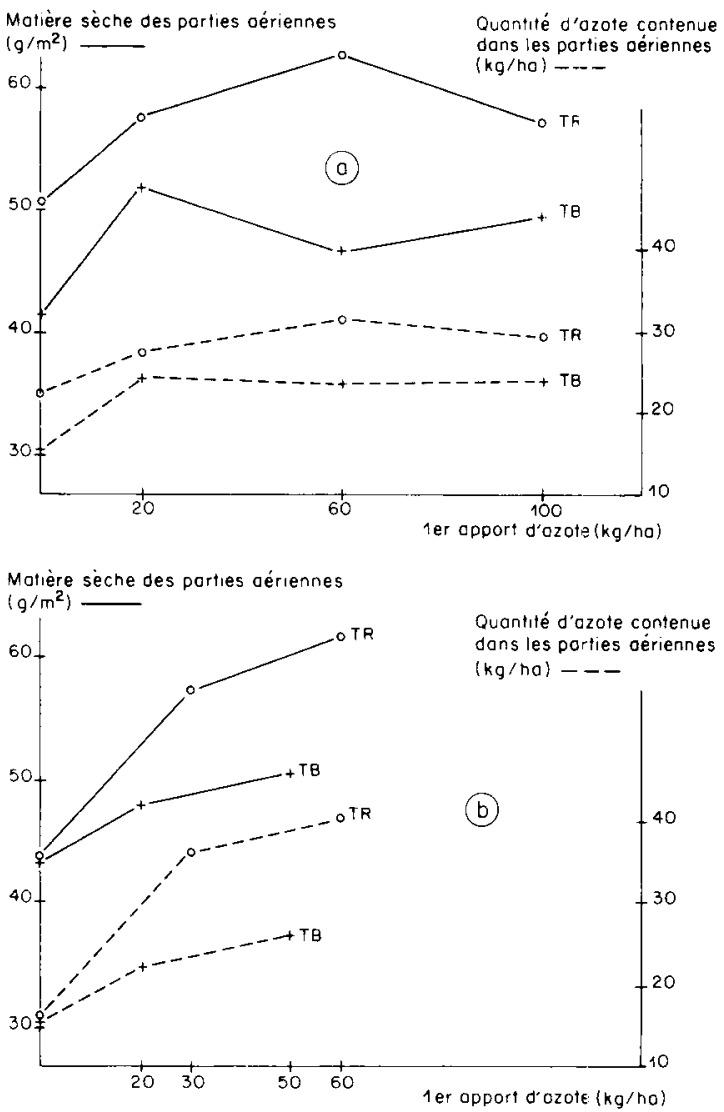

Figure 2

Type de sol et réponse à l'azote de la croissance des parties aériennes (mesurées au stade $B_{I I}$ ) en 1977 (a) et 1978 (b).

$T B=$ Terre Blanche $; T R=$ Terre Rouge.

Soil classes and aerial growth response to nitrogen at JONARD $B_{I I}$ stage in $1977(a)$ and $1978(b) . T B=$ White soil ; TR $=$ Red soil. -.-- Nitrogen content in aerial parts ( $\mathrm{kg} / \mathrm{ha}$ ).

- Dry matter in aerial parts $\left(\mathrm{g} / \mathrm{m}^{2}\right)$.

TABLEAU 5

Effet du type de sol (Terre blanche: TB ou Terre rouge: TR) sur le peuplement-épi (en nombre d'épis/m²).

Effect of soil class on ear density (ear number per square meter). TB: "White soils " class - TR : "Red soils " class.

\begin{tabular}{|c|c|c|c|c|c|c|}
\hline \multirow{2}{*}{ Campagne } & \multirow{2}{*}{$\begin{array}{l}\text { Variétés } \\
\text { (nombre de } \\
\text { couples) }\end{array}$} & \multicolumn{2}{|c|}{ Valeurs moyennes } & \multirow{2}{*}{$\begin{array}{c}\text { Test } \\
\text { global } \\
\text { des couples }\end{array}$} & \multicolumn{2}{|c|}{ Nombre de cas où : } \\
\hline & & $\mathrm{TB}$ & TR & & $\begin{array}{r}\mathrm{TB}>\mathrm{TR} \\
\text { (test de }\end{array}$ & $\begin{array}{c}\mathrm{TB}<\mathrm{TR} \\
\mathrm{P}=0,10)\end{array}$ \\
\hline \multirow[b]{2}{*}{1975} & Joss (7) & 362 & 418 & \multirow[b]{2}{*}{$S(P=0,05)$} & \multirow[b]{2}{*}{$1 / 10$} & \multirow[b]{2}{*}{$3 / 10$} \\
\hline & TALENT (3) & & & & & \\
\hline \multirow[b]{2}{*}{1976} & TALENT (3) & 524 & 594 & \multirow[b]{2}{*}{$S(P=0,10)$} & \multirow[b]{2}{*}{$1 / 10$} & \multirow[b]{2}{*}{$5 / 10$} \\
\hline & TOP (7) & 448 & & & & \\
\hline \multirow[b]{2}{*}{1977} & TALENT (7) & 616 & 698 & \multirow[b]{2}{*}{$S(P=0,02)$} & \multirow[b]{2}{*}{0} & \multirow[b]{2}{*}{$4 / 10$} \\
\hline & TOP (2) & 523 & 548 & & & \\
\hline
\end{tabular}


Pour les traitements ayant reçu de fortes doses au tallage, l'azote n'a pas été facteur limitant avant le stade B et la Terre rouge manifeste une nette supériorité dans ces conditions. De ce fait, et malgré une croissance plus forte en l'absence d'engrais, la réponse aux apports tallage y est plus marquée qu'en Terre blanche. Sur un essai analogue, réalisé en 1978 (fig. $2 b$ ), on observe les mêmes tendances.

En Terre blanche, se manifeste un facteur (ou condition) limitant(e) qui n'est ni l'azote, ni l'eau. Or jusqu'au stade B, le sol est peu couvert et les conditions de milieu correspondent bien à celles qui ont été énoncées précédemment pour que s'exprime une différence de température de surface entre Terre rouge et Terre blanche (c'est précisément des essais EEB et EER que proviennent les résultats du tableau 3). Il est probable que ce sont ces différences de températures qui expliquent les variations de croissance maximum entre les 2 types de sol.

On peut penser qu'elles ont également une influence sur la progression de l'enracinement en profondeur. En 1976 et 1977, des observations d'enracinement ont été réalisées au stade B : on constate que les profondeurs atteintes par les racines en stations de Terre rouge sont, sauf lorsqu'il s'y trouve un encroûtement à faible profondeur, toujours supérieures ou égales à celles atteintes dans les stations de Terre blanche (comparaisons en couples).

En définitive, les différences du nombre d'épis à l'avantage des Terres rouges, constatées au paragraphe précédent, peuvent s'expliquer conjointement :

- par l'effet direct de la température sur la croissance des parties aériennes. En effet, J. MASLE-MEYNARD (1980) a montré que la masse de matière sèche obtenue en début de montaison était un bon prédicteur du nombre d'épis ;

- par une exploitation plus précoce des réserves d'azote minéral par les racines.

On ne peut exclure l'hypothèse supplémentaire d'une minéralisation plus précoce.

\section{Conséquences pour les modalités d'apport des engrais azotés}

\section{a) Apport tallage}

Sur tous les essais de fumure azotée effectués en Terre blanche lors des 3 années d'étude, on a constaté que le nombre d'épis n'était pas influencé par la quantité d'azote apportée au tallage. Ceci correspond bien à l'absence de réponse de la croissance des parties aériennes jusqu'au stade $B_{11}$, mise en évidence dans le paragraphe précédent.

Ainsi, en Terre blanche, la croissance précoce des pieds de blé étant limitée par la température, on ne peut espérer influer sur le nombre d'épis que par l'intermédiaire de la densité de semis et, peut-être, la date de semis.

En Terre rouge, on attend une réponse plus marquée $d u$ nombre d'épis aux apports précoces. On peut donc espérer valoriser des doses d'engrais plus élevées.

\section{b) Date du second apport}

A partir du stade $B_{I 1}$ (épi à $1 \mathrm{~cm}$ du plateau de tallage), l'absorption d'azote par le peuplement végétal s'accélère. C'est donc à ce stade qu'il est recommandé d'effectuer le $2^{\mathrm{e}}$ apport d'engrais, afin d'éviter que l'alimentation azotée ne risque de limiter la croissance, même temporairement.

A cet égard, on peut considérer que les Terres rouges permettent à l'agriculteur une plus grande souplesse que les Terres blanches: en effet, l'absence temporaire d'engrais peut être compensée en Terre rouge par une exploitation plus profonde des reliquats d'azote ct, sans doute, par unc minćralisation plus précoce.

Cette conclusion s'est trouvée vérifiée en 1975: les instructions concernant les stades de réalisation du second apport sur les parcelles-types ayant été plus ou moins bien suivies, il en est résulté un étalement des stades d'apport, du stade $B_{11}$ au stade $C_{\mathrm{II}}$ (définitions de JONARD et al., 1952). La position respective des stades de la plante et de l'apport d'engrais peut ĉtre paramétrée par la somme de degrésjours comptée du semis au jour de l'apport, soit $\mathrm{S}$ ce paramètre.

On constate que la différence de nombre de grains par $\mathrm{m}^{2}$ entre les 2 membres d'un couple Terre rouge-Terre blanche (soit DNG) croît au détriment de la station Terre blanche, lorsqu'augmente le retard de l'apport par rapport au stade jugé optimal ; on obtient la relation statistique :

$\mathrm{DNG}=0,81 \mathrm{~S}-61,8 \quad($ avec $\mathrm{r}=0,73$, pour 9 couples, significatif à 0,05 ).

\section{Influence sur l'alimentation hydrique, choix d'un rendement-objectif}

Au cours de la montaison du blé, sous l'influence d'un déficit climatique généralement croissant, le sol s'assèche progressivement. L'alimentation hydrique peut alors devenir limitante et, dans ce cas, la croissance dépend des réserves du sol et de leur disponibilité, dont on a souligné plus haut la grande variabilité dans le milieu considéré.

Ainsi, en 1976, on a observé une relation étroite entre les poids de 1000 grains et la colonisation par les racines des horizons de craie (SEBILlotTE et al., 1978).

Dans des conditions de fort déficit climatique, ce sont donc les réserves du sol qui déterminent le rendement maximum que l'on peut obtenir sur une parcelle.

Sur la figure 3 , on a représenté, en fonction du bilan ETP-

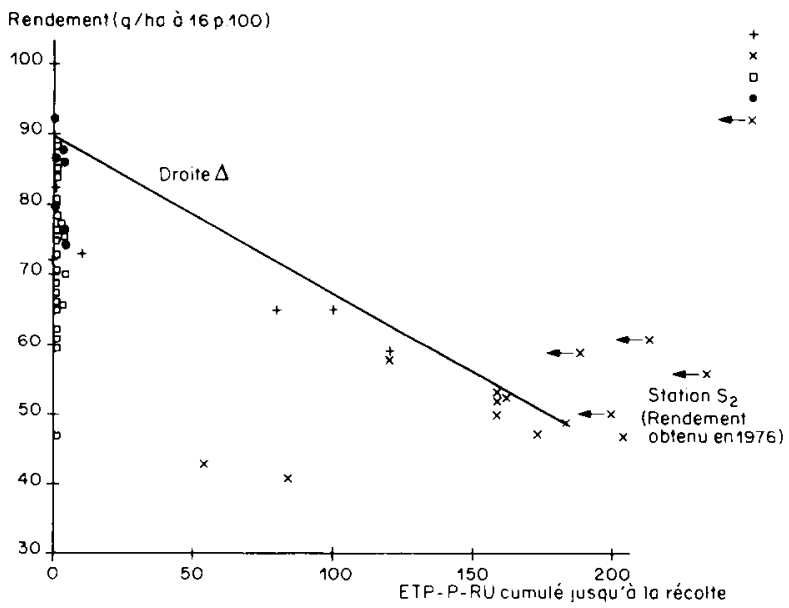

Figure 3

Rendement des stations suivies en Champagne crayeuse 4 années consécutives, en fonction du bilan hydrique (variété "Talent").

Yields obtained on experimental plots for 4 years in Champagne Crayeuse, in relation to water balance.

$\left.\begin{array}{cc}+ & 1975 \\ \times & 1976 \\ - & 1977 \\ - & 1978\end{array}\right\}$ année d'observation

$-\times$ Station dont la réserve utile a vraisemblablement été sous-estimée (les racines entrent au contact de la craie, sans la coloniser). - Experimental plot whose available water content was probably under-estimated. 
P-RU (cumulé à partir du début d'épuisement de la réserve, situé généralement aux alentours de début avril, jusqu'à la récolte), les rendements obtenus sur les stations de variété « Talent " lors des différentes campagnes d'étude. On observe que les rendements maxima atteints pour un déficit de bilan donné sont d'autant plus faibles que ce déficit est important, presque tous les points sont situés en dessous d'une limite dont la position peut être représentée approximativement par une droite $\Delta$ d'équation:

$$
\mathrm{R} \max =90-0,23 \text { (ETP-P-RU). }
$$

Au-dessus de cette droite ne se trouvent que les points représentatifs des stations où les racines entrent en contact avec la craie sans la coloniser, et dont on sous-estime la réserve utile (cas de $S_{2}$ du tableau 4). Bien entendu, la fixation de cette équation (qui est ici simplement graphique et ne résulte pas d'un calcul de régression, s'agissant d'une limite supérieure) est assez arbitraire, vu le nombre de points relativement restreint. Il serait donc nécessaire de l'affiner par des résultats supplémentaires; néanmoins, nous l'utiliserons ci-dessous pour donner un support concret au raisonnement.

En effet, ces résultats permettent d'envisager de façon rationnelle le choix d'un objectif de rendement, nécessaire pour la détermination de la fumure azotée d'après le bilan prévisionnel.

L'étude des déficits climatiques des années 1950 à 1979 montre que :

- 80 p. 100 des déficits climatiques (ETP-P) sont supérieurs à $100 \mathrm{~mm}$;

- 50 p. 100 sont supérieurs à $190 \mathrm{~mm}$;

- 20 p. 100 sont supérieurs à $240 \mathrm{~mm}$.

Si l'on admet :

- qu'il est inutile de viser un rendement ayant moins de 50 p. 100 de chances d'être réalisé,

- et qu'inversement, il faut restreindre à 50 p. 100 le risque de limiter le rendement par défaut d'azote,

on retient alors le déficit médian de $190 \mathrm{~mm}$. Bien entendu, d'autres choix de risques sont possibles. Dans l'hypothèse retenue, la figure 4 nous permet, pour la variété «Talent ", et sous réserve que les autres facteurs limitants possibles (parasitisme, bases échangeables, cf. MEYNARD et al., 1981) soient levés, de poser l'objectif de rendement à :

- $90 \mathrm{q} /$ ha pour une Terre blanche analogue à la station R1 du tableau $4(\mathrm{RU}=260 \mathrm{~mm})$.

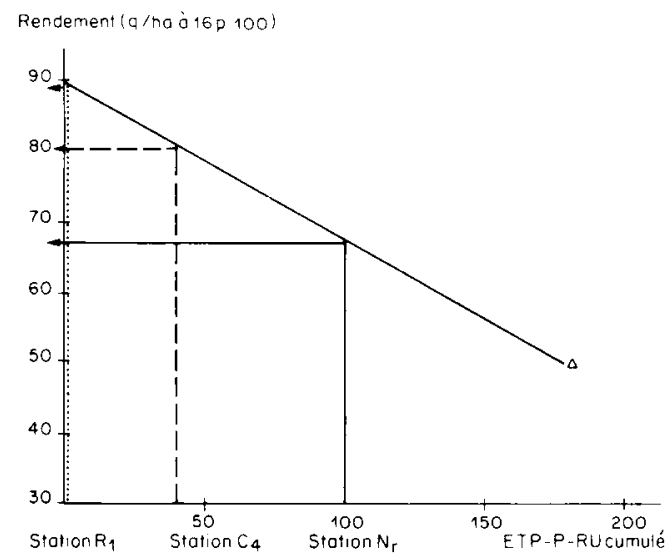

Figure 4

Choix des objectifs de rendement pour trois stations.

Determination of the objective yields for three plots.
- $80 \mathrm{q} / \mathrm{ha}$ pour une Terre rouge analogue à $\mathrm{C} 4(\mathrm{RU}=$ $150 \mathrm{~mm})$.

- $68 \mathrm{q} /$ ha pour une graveluche encroûtée de type $\mathrm{N}_{\mathrm{r}}$ $(\mathrm{RU}=90 \mathrm{~mm})$.

\section{CONNAISSANCE DES SOLS ET CONDUITE DE LA CULTURE}

En Champagne crayeuse, la diversité pédologique se traduit par de grandes variations des états structuraux du sous-sol, ainsi que par des différences importantes de constitution physicochimique de la couche arable (teneur en calcaire et en éléments grossiers).

Il en résulte des différences:

- de profondeur d'enracinement, donc de possibilité d'exploitation des réserves en eau et en azote minéral par le peuplement végétal ;

— de régime thermique, donc de vitesse de croissance des parties aériennes et des racines jeunes.

- et, peut-être, de minéralisation de l'azote organique due à la fois aux variations de teneur en calcaire actif, de régime thermique et de teneurs en matière organique.

La classification vernaculaire, utilisée par les agriculteurs, traduit de façon correcte les principales différences de comportement du sol et de la végétation, mais elle est peu nuancée.

Or, les modalités de conduite de la culture, et tout particulièrement la mise en ouvre de la fertilisation azotée, doivent être adaptées, de façon relativement précise, aux caractères pédologiques de la parcelle. En effet, ceux-ci influencent certains termes du bilan prévisionnel (objectif de rendement, reliquat d'azote minéral en sortie d'hiver, minéralisation) et doivent être pris en compte pour l'application des engrais (fractionnement et dates d'apport); ils ont aussi des répercussions sur le choix des dates et doses de semis ou de variétés. Par exemple, on recommandera des variétés précoces et des semis avancés en sol de faible réserve hydrique pour diminuer les risques d'échaudage ; en Terre blanche, on conseillera des semis denses, seule façon d'obtenir des peuplements-épis élevés en l'absence de réponse à l'azote apporté au tallage.

L'élaboration de tels conseils exige donc une connaissance pédologique approfondie. On peut considérer que le niveau de précision requis correspond sensiblement à celui atteint dans la description d'une série pédologique.

Cependant, les catégories constituant des unités de conseil agronomique ne correspondent pas nécessairement aux unités pédogénétiques de niveau supérieur. Dans le cas étudié de la conduite du blé, il convient de regrouper les types de profil élémentaires en fonction, d'une part du comportement thermique, d'autre part de la réserve hydrique.

Au sein du tableau 1 , on est donc amené à distinguer les sols où la craie est présente dans le profil cultural, en raison des importantes conséquences physiques qui en découlent. Ce premier critère de distinction oppose les catégories (1) à (5) de ce tableau aux autres catégories.

- Parmi les sols présentant de la craie dans le profil, la proximité immédiate de celle-ci avec la couche arable constitue un élément important de distinction des comportements thermiques et des probabilités d'accès des racines à la craie. On définit donc 2 unités, respectivement : I (catégories (1), (2), (3)) et II (catégories (4) et (5)). 


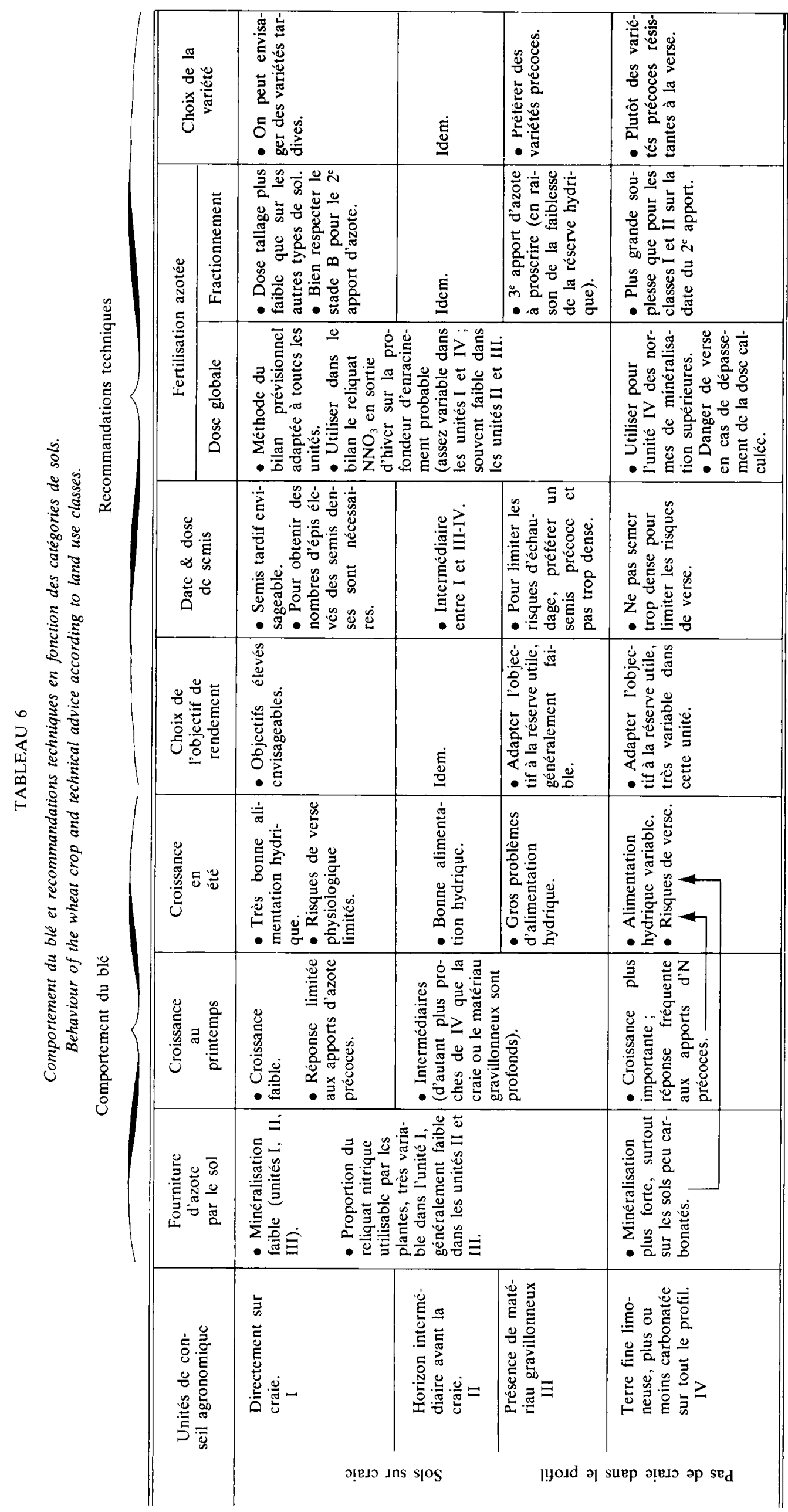


- Parmi les sols sans craie dans le profil, on doit distinguer 2 unités :

- ceux formés sur colluvions hérités de la craie, le plus souvent gravillonneux et encroûtés, correspondant à une réserve en eau particulièrement faible (catégories (6), (7), (8) et (9), formant l'unité III) ;

- ceux, généralement plus profonds, où le substrat dominant est la terre fine, plus ou moins carbonatće (catégories (10) et (11), formant l'unité IV).

A chacune de ces grandes unités, correspondent des références sur le comportement du blé et les recommandations techniques qui s'en déduisent. Elles sont présentées dans le tableau 6.

Une des limites dans l'application, déjà entreprise, de ces recommandations, réside dans la façon dont ont été constituées les parcelles. Un grand nombre d'entre elles sont très grandes, ou mal disposées par rapport à la toposéquence et présentent, en leur sein, des zones appartenant à des catégories contrastées. Néanmoins, certaines variations dans les réglages des outils doivent rester possibles dans de nombreux cas.

\section{CONCLUSION}

Le travail présenté dans ce mémoire illustre la complémentarité et les liens nécessaires entre l'approche pédologique à grande échelle et le diagnostic agronomique.
A partir de l'étude de nombreuses stations d'observation en Champagne crayeuse, on a confirmé la forte variabilité pédologique de cette région et analysé, dans son contexte climatique, les répercussions de cette variabilité sur l'élaboration du rendement du blé d'hiver.

On en déduit un ensemble d'indications sur l'emploi des engrais azotés et des prescriptions de conduite de la culture, qui représentent le "mode d'emploi » de la méthode du bilan prévisionnel au niveau de la parcelle. Ces conseils sont formulés par grande unité de milieu: celles-ci, regroupant les unités pédologiques de base sur des critères agronomiques, constituent une clé d'extrapolation régionale des conclusions de l'étude.

A ce titre, elle devient un guide pour les agents du développement: elle leur permet de substituer à une appréciation empirique de la parcelle une prise en compte objective des caractères de celle-ci, y compris ses potentialités de rendement.

Reçu le 5 mars 1981. Accepté le 16 décembre 1981.

\section{REMERCIEMENTS}

Nous remercions MM. Durand, Dutil, Muller et Vachier de la Station de Science du Sol de Chalons, pour le précieux concours qu'ils nous ont apporté dans la connaissance des sols de Champagne crayeuse, ainsi que les agriculteurs du CETA de Romilly et son ingénicur, M. FERRATON, pour leur coopération active.

\section{ANNEXE 1}

Evaluation des réserves utiles des principaux matériaux observés.

Evaluation of available water in the principal materials investigated.

\begin{tabular}{|c|c|c|c|c|c|c|c|c|c|}
\hline \multirow{3}{*}{ Horizon } & \multicolumn{6}{|c|}{ Gamme d'eau utile } & \multirow[b]{2}{*}{$\begin{array}{l}\text { Valeur } \\
\text { retenue }\end{array}$} & \multirow{3}{*}{$\begin{array}{c}\text { Densité } \\
\text { apparente }\end{array}$} & \multirow{3}{*}{$\begin{array}{c}\text { R.U. } \\
\text { (mm par dm } \\
\text { d'horizon) }\end{array}$} \\
\hline & \multicolumn{3}{|c|}{$\begin{array}{l}\text { Borne supéricure } \\
\text { pour } 100 \text { de terre sèche }\end{array}$} & \multicolumn{3}{|c|}{$\begin{array}{l}\text { Borne inféricure } \\
\text { pour } 100 \text { de terre sèche }\end{array}$} & & & \\
\hline & $\begin{array}{c}\text { Humidité } \\
\text { equivalente } \\
\text { (l) }\end{array}$ & $\begin{array}{l}\text { Valcurs } \\
\text { au champ } \\
\left({ }^{2}\right)\end{array}$ & $\begin{array}{c}\text { Valcurs } \\
\text { de BALLIF } \\
(3)\end{array}$ & $\begin{array}{c}\mathrm{H}_{\mathrm{pF}+4.2} \\
(+)\end{array}$ & $\begin{array}{c}\text { Valcurs } \\
\text { au champ } \\
(5)\end{array}$ & $\begin{array}{c}\text { Valcurs } \\
\text { de BALLII: } \\
\left({ }^{(\prime)}\right)\end{array}$ & p. 100 TS & & \\
\hline Couche arable Terre blanche & 26 & 25 & \multirow{2}{*}{$20.7-21,5$} & 9 & 5 & \multirow{2}{*}{$10,6-11.5$} & 16 & 1,25 & 20 \\
\hline Couche arable Terre rouge & 24 & 24 & & 10 & 6 & & 14 & 1.25 & 18 \\
\hline \multirow{2}{*}{ Craic $\begin{array}{l}\text { remanićc } \\
\text { cn place }\end{array}$} & 24 & 22 & 25,7 & - & 4 & $2,5-2,6$ & 20 & 1,25 & 25 \\
\hline & 24 & 22 & $25,0-26.9$ & 5 & - & $1,2-1,7$ & 20 & 1,40 & 28 \\
\hline Terre fine limoneuse & 25 & 23 & - & - & 6 & - & 16 & 1,35 & 22 \\
\hline \multicolumn{10}{|l|}{ Matériau gravillonncux } \\
\hline$>50 \%$ gravillons & - & 19 & 17,7 & - & 6 & 2,3 & 12 & 1,30 & 16 \\
\hline dc 25 à $50 \%$ gravillons & 29 & 26 & - & - & - & - & 14 & 1,35 & 19 \\
\hline \multicolumn{10}{|l|}{ Palćosol } \\
\hline Poches de cryoturbation & 19 & 17 & $17.5-17.8$ & - & 4 & $3.5-5.0$ & - & - & - \\
\hline Cheminées & \multirow{2}{*}{\multicolumn{6}{|c|}{ (cf. Craic remanićc) }} & & & \\
\hline Ensemble de l'horizon & & & & & & & 15 & 1,35 & 20 \\
\hline
\end{tabular}

(1) Laboratoire d'Arras. Valcurs moyennes obtenues à partir de quelques profils-types ;

(') Observations au champ en sortic d'hiver et début de printemps; valeurs moyennes;

(3) Extraits de BALLIF, 1980. Valcurs observées au champ sur 2 profils suivis 4 ans :

(4) Déterminations sur 1 ou 2 échantillons-types (extracteur à plaque);

(5) Moyennes d'observations réalisćes en août 1976 ;

(6) Valeurs de $\mathrm{H}_{\mathrm{pF} 4.2}$, déterminées par BALLIF sur 2 profils-types:

(7) Valeurs choisies d'après BALLIF, déjà cité. 


\section{RÉFÉRENCES BIBLIOGRAPHIQUES}

Ballif J. L., 1978. Porosité de la craie. Appréciation de la taille et de la répartition des pores. Ann. agron., 29 (2), 123-131.

Ballif J. L., 1980. Caractères et réserves hydriques des sols sur craie et sur graveluche en Champagne. Ann. agron., 31 (4), 473-485.

Ballif J. L., 1981. Comportement hydrique d'une rendzine brune sur craie sous l'influence de la sécheresse. C.R. Acad. Agric. Fr., 67 (5), 395-403.

Ballif J. L., Dutil P., 1974. Utilisation des films plastiques photodégradables pour la culture du mais en Champagne crayeuse. C.R. Acad. Agric. Fr., 60 (6), 389-401.

Ballif J. L., Dutil P., 1975. Le réchauffement des sols de craie par films plastiques. Mesures et bilans thermiques. Ann. agron., 26 (2), 159-167.

Boiffin J., Caneill J., Meynard J. M., Sebillotte M., 1981. Elaboration du rendement et fertilisation azotée du blé d'hiver en Champagne crayeuse. I - Protocole et méthode d'étude d'un problème technique régional. Agronomie, 1 (7), 549-558.

Durand R., 1972. Les sols sur craie. Régions de Breban-Chapclaine (Marne) et de Dampierre (Aube). Sci. Sol, (6), 269-287.

Durand R., 1980. L'évolution d'une rendzine encroûtée sur la craic de Champagne. Sci. Sol, (3), 201-216.

Dutil P., 1970. Caractères généraux des sols de Champagne crayeuse. $95^{e}$ Congrès des Sociétés Savantes, Reims, 1970, 9 p.

Dutil P., Ballif J. L., 1968. Sur la présence fréquente en Champagne crayeuse de rendzines développécs sur paléosols cryoturbés. Sci. Sol, (2), 79-91.

Dutil P., Ballif J. L., 1971. Prévision de la fumure azotée du blé d'hiver en Champagne crayeuse. C.R. Acad. Agric. Fr., 57 (1), 8895.

Gachon L., 1973. Influence du système cultural sur l'économie de l'azote en sol argilo-calcaire de Limagne. Sci. Sol, (3), 177-197.

Gras R., 1972. Effets des éléments grossiers sur la dynamique de l'eau dans un sol sableux (I). Ann. agron., 23 (2), 197-239.
Gras R., Monnier G., 1963. Contribution de certains éléments grossiers du sol à l'alimentation en eau des végétaux. Sci. Sol, (1), 13-30.

Hébert J., 1969. La fumure azotée du blé tendre d'hiver. Bull. Techn. Inf. Min. Agric., (244), 755-766.

Jonard P., Koller J., Vincent A., 1952. Evolution de la tige et de l'épi chez la variété de blé "Vilmorin 27 » au cours de la période de reproduction. Ann. Amélior. Plant., 2 (1), 31-54.

Lindemann Y., 1977. Conséquences sur le plan agronomique de l'hétérogénéité des répartitions de l'Azote nitrique et du Potassium dans les sols. In : "Le Potassium en agriculture 》, CAPILlON A., Sebillotte M., Doc. INA-PG, Paris, 213 p., 42-57.

Masle-Meynard J., 1980. L'élaboration du nombre d'épis chez le blé d'hiver. Influence de différentes caractéristiques de la structure du peuplement sur l'utilisation de l'azote et de la lumière. Thèse de Docteur Ingénieur, Paris, 201 p. + Annexes.

Meynard J. M., Boiffin J., Caneill J., Sebillotte M., 1981. Elaboration du rendement et fertilisation azotée du blé d'hiver en Champagne crayeuse. II - Types de réponse à la fumure azotée et application de la méthode du bilan prévisionnel. Agronomie, 1 (9), 795-806.

Muller J., 1977. Situation des réserves en azote nitrique dans les sols de Champagne crayeuse à la sortie de l'hiver 1976-77. I.N.R.A. Chalons, Doc. Ronéo., 11 p.

Pédro G., 1972. Les sols développés sur roche calcaire. Sci. Sol, (1), 5-18.

Rémy J. C., Hébert J., 1977. Le devenir des engrais azotés dans le sol. C.R. Acad. Agric. Fr., 63 (11), 700-710.

Roncin F., 1978. Le réchauffement des sols de craie. Relation entre l'évaporation du sol nu et ses caractéristiques physiques. Chairc d'Agronomie, INA-PG, Paris, Doc. Ronćo., 23 p.

Sebillotte M., Boiffin J., Caneill J., Meynard J. M., 1978. Sécheresse et fertilisation azotée du blé d'hiver. Essai d'analyse de situations au champ par l'étude des composantes du rendement. Sci. Sol, (3), 197-214. 